Prepared for the U.S. Department of Energy under Contract DE-AC05-76RL01830

\title{
Final Report for Monitoring of Reactor Antineutrinos with Compact Germanium Detectors
}

JL Orrell

JI Collar

July 2009

Pacific Northwest

NATIONAL LABORATORY

Proudly Operated by Battelle Since 1965 


\title{
DISCLAIMER
}

This report was prepared as an account of work sponsored by an agency of the United States Government. Neither the United States Government nor any agency thereof, nor Battelle Memorial Institute, nor any of their employees, makes any warranty, express or implied, or assumes any legal liability or responsibility for the accuracy, completeness, or usefulness of any information, apparatus, product, or process disclosed, or represents that its use would not infringe privately owned rights. Reference herein to any specific commercial product, process, or service by trade name, trademark, manufacturer, or otherwise does not necessarily constitute or imply its endorsement, recommendation, or favoring by the United States Government or any agency thereof, or Battelle Memorial Institute. The views and opinions of authors expressed herein do not necessarily state or reflect those of the United States Government or any agency thereof.

\author{
PACIFIC NORTHWEST NATIONAL LABORATORY \\ operated by \\ BATTELLE \\ for the \\ UNITED STATES DEPARTMENT OF ENERGY \\ under Contract DE-AC05-76RL01830
}

Printed in the United States of America

Available to DOE and DOE contractors from the

Office of Scientific and Technical Information,

P.O. Box 62, Oak Ridge, TN 37831-0062;

ph: (865) 576-8401

fax: $(865) 576-5728$

email: reports@adonis.osti.gov

\footnotetext{
Available to the public from the National Technical Information Service, U.S. Department of Commerce, 5285 Port Royal Rd., Springfield, VA 22161 ph: (800) 553-6847 fax: $(703) 605-6900$

email: orders@ntis.fedworld.gov

online ordering: http://www.ntis.gov/ordering.htm
}

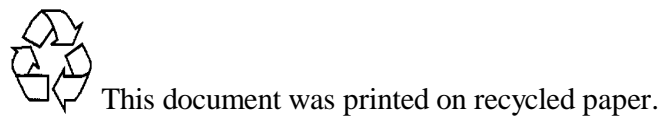




\section{Final Report for Monitoring of Reactor Antineutrinos with Compact Germanium Detectors}

JL Orrell

Jl Collar

July 2009

Prepared for

the U.S. Department of Energy

under Contract DE-AC05-76RL01830

Pacific Northwest National Laboratory

Richland, Washington 99352 


\section{Summary}

This 2008 National Consortium for Measures and Signatures Intelligence (MASINT) Research (NCMR) project has pursued measurement of the antineutrino-nucleus coherent scattering interaction using a lowenergy threshold germanium gamma-ray spectrometer of roughly one-half kilogram total mass. These efforts support development of a compact system for monitoring the antineutrino emission from nuclear reactor cores. Such a monitoring system is relevant to nuclear safeguards and nuclear non-proliferation in general because it adds a strong method for assuring quantitative material balance of special nuclear material in the nuclear fuel cycle used in electricity generation.

Findings of the work partially supported by the NCMR program include:

- Present low-energy threshold germanium detectors operated at a nuclear reactor show an energy threshold of $500 \mathrm{eV}$.

- Laboratory operation has demonstrated a best energy threshold of $300 \mathrm{eV}$ with this detector. This is more than an order of magnitude below the low-energy threshold of comparable mass detectors.

- The threshold (300 - $500 \mathrm{eV})$ is not low enough to measure antineutrino-nucleus coherent scattering.

- Efforts to reduce the threshold to the $50-100 \mathrm{eV}$ level achieved with X-ray detectors have demonstrated a consistent $500 \mathrm{eV}$ threshold.

- The continuum background - a consequence of cosmic-ray activation - is at the level of 20 50 counts/keV/kg/day.

- The continuum background achieved at the shallow depth of 30 meters of water equivalent depth is comparable to deep underground experiments despite a factor of 25,000 greater cosmic-ray rate.

Conclusions:

1. A first reactor measurement has been made using a low-energy threshold germanium detector.

i. The detector is not yet capable of measuring antineutrino-nucleus coherent scattering.

ii. The present shield design and background level are adequate.

2. Future effort must focus on reducing the electronics noise to achieve a $50-100 \mathrm{eV}$ energy threshold that would allow measurement of antineutrino-nucleus coherent scattering.

Recommendation:

Refocus research efforts on reduction of the germanium detector threshold. This is a laboratory effort done away from a reactor site. There are two prongs to this effort:

1. Selecting and testing electronics components for low noise performance.

2. Modifying the mounting materials to limit dielectrically induced noise. 


\section{Acronyms and Abbreviations}

$\begin{array}{ll}\text { CDMS } & \text { Cryogenic Dark Matter Search } \\ \text { CGS } & \text { Columbia Generating Station } \\ \text { GRETINA } & \begin{array}{l}\text { GRETINA is the initial stage effort of the full Gamma-Ray Energy Tracking Array } \\ \text { (GRETA) experiment. } \\ \text { IC }\end{array} \\ \text { LLNL } & \text { Intelligence Community } \\ \text { MARS R\&D } & \text { Multi-sensor Airborne Radiation Survey Research and Development } \\ \text { PMP } & \text { Project Management Plan } \\ \text { PNNL } & \text { Pacific Northwest National Laboratory } \\ \text { SNL } & \text { Sandia National Laboratory } \\ \text { SONGS } & \text { San Onofre Nuclear Generating Station } \\ \text { SSI } & \text { Safeguards Summer Internship } \\ \text { UV } & \text { Ultraviolet }\end{array}$




\section{Contents}

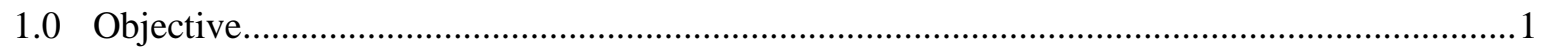

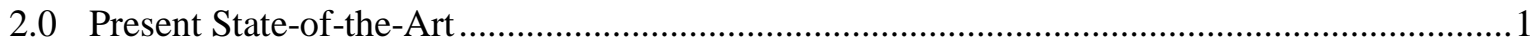

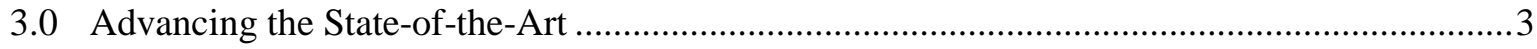

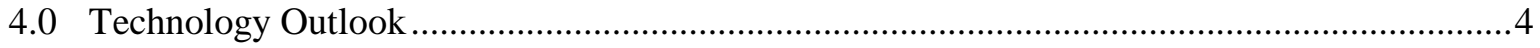

Appendix A : Work completed by Task from the Statement of Work ........................................A-1

Appendix B : Work leveraged from other projects .................................................................. B-1

Appendix C : Program development........................................................................................ 


\subsection{Objective}

The objective of this NCMR project is to measure the antineutrino-nucleus coherent scattering crosssection. This interaction process is anticipated by the Standard Model of particle physics but has yet to be experimentally confirmed because of the technological difficulty in building a detector having simultaneous large mass $(\sim 1 \mathrm{~kg})$ and low energy threshold $(\sim 100 \mathrm{eV})$. This project is pursuing development of such a detector.

The measurement objective of this project is relevant to nuclear safeguards and nuclear non-proliferation in general. Measurement of the antineutrino-nucleus coherent scattering cross-section opens the way to using this interaction mechanism to develop a method for monitoring the antineutrino emission from an operating nuclear reactor core. The antineutrinos that free-stream out of the reactor core (and building) are tied to the total nuclear fission rate of the reactor core. On average, each nucleus that undergoes fission leads to six emitted antineutrinos as the unstable fission products decay towards stability. Thus, the ability to count the number of emitted antineutrinos provides an independent measure of the total fission rate of the reactor core. This information provides a near real-time point of comparison for calculations of the material balance of uranium input fuel and produced plutonium.

Although not part of the scope of this NCMR project, the successful measurement of the antineutrinonucleus coherent scattering cross-section creates a straightforward (though difficult) route to building a demonstration germanium-based reactor monitoring system. For clarity of long-term research objectives, such a system is referenced throughout this report as the end-product of the research program that this NCMR project initiates.

\subsection{Present State of the Art}

The general concept of measuring the antineutrino emission from an operating reactor core is not a new reactor monitoring concept. Lawrence Livermore National Laboratory (LLNL) in collaboration with Sandia National Laboratory (SNL) has developed an antineutrino monitoring apparatus using liquid scintillator as the primary detection medium. Figure 1 shows a design cut-away of the LLNL/SNL liquid scintillator-based detector technology. 


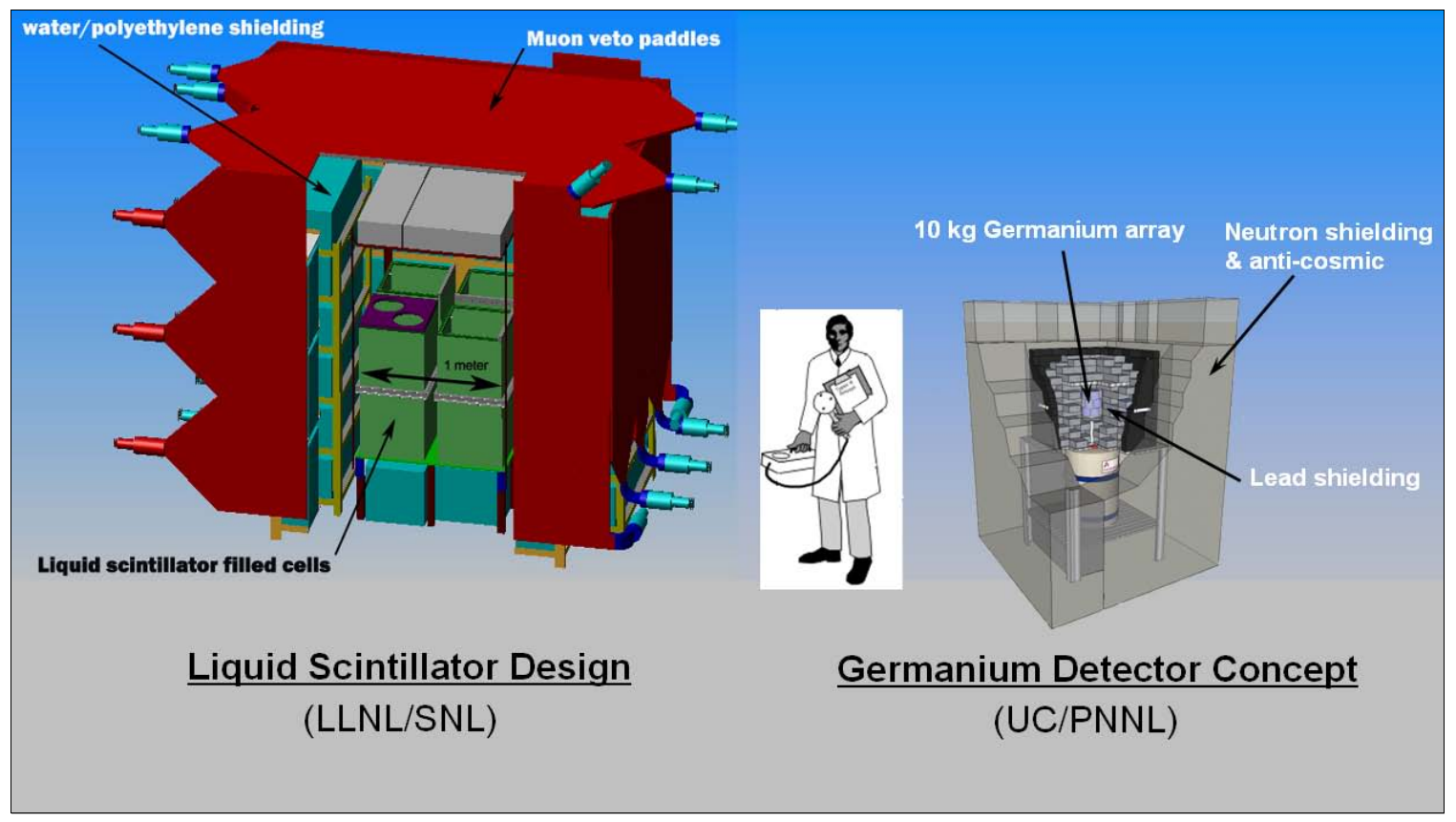

Figure 1 - The LLNL scintillator-based antineutrino monitor (left) and the concept for a future germanium-based antineutrino monitor (right), roughly a factor of five less volume. Each instrument is shown in comparison to human scale.

The scintillator-based antineutrino monitoring concept operates on the principle of proton absorption of antineutrinos. That is, protons contained in the liquid scintillator medium absorb antineutrinos emitted from the reactor core and together transform into positrons and neutrons with a 1.8-MeV reaction threshold:

$$
\bar{v}+p \rightarrow e^{+}+n-1.8 \mathrm{MeV}
$$

This method of detecting antineutrinos is the same as used by F. Reines and C.L. Cowen in 1956 to confirm the existence of the antineutrino, leading to the award of the 1995 Nobel Prize in Physics to F. Reines.

The LLNL technology clearly demonstrates the viability of using antineutrinos to monitor reactor core activity as demonstrated by the results presented in Figure 2. 


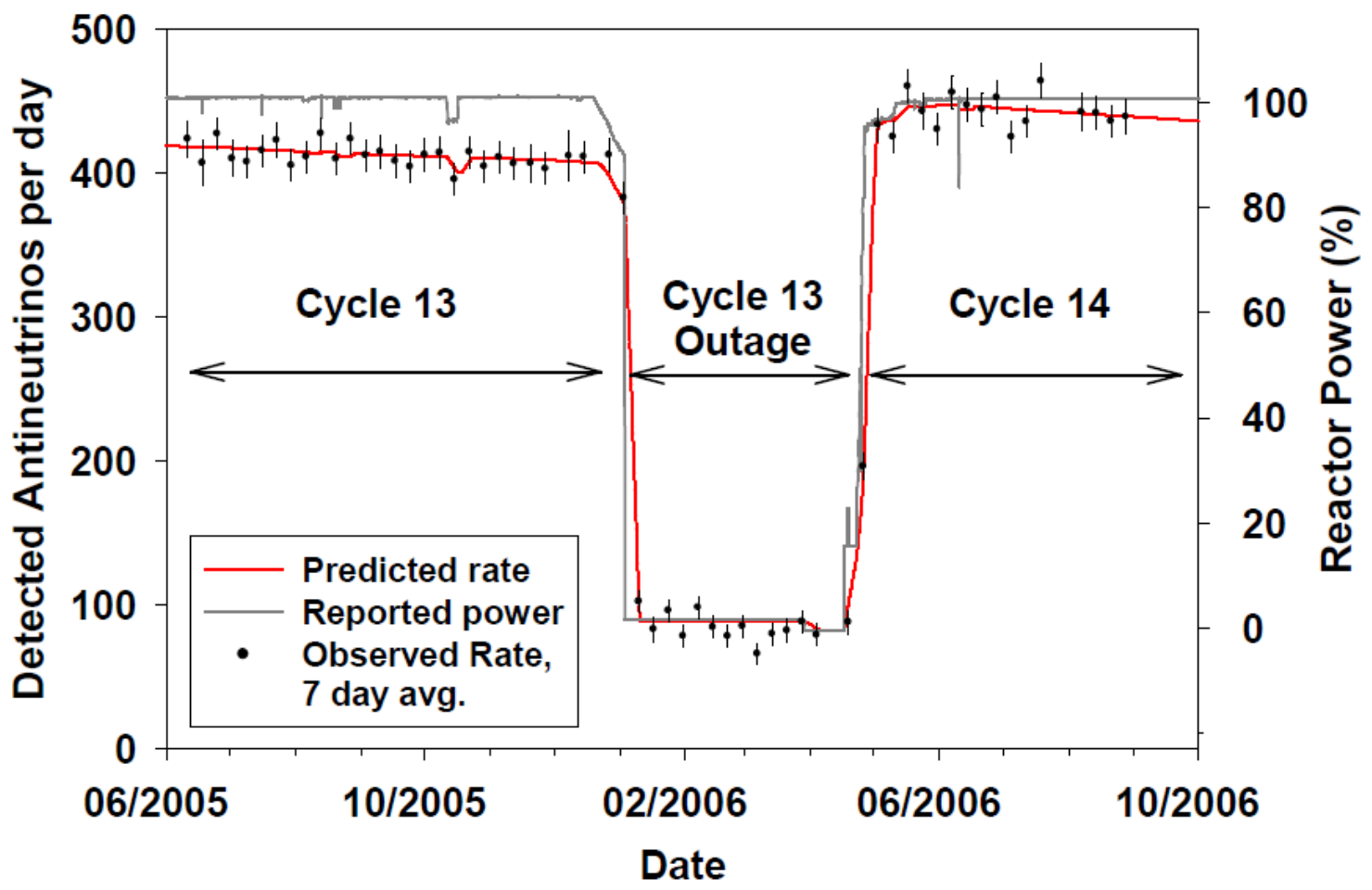

Figure 2 - Results from the LLNL/SNL liquid scintillator-based antineutrino detector show its ability to measure the relative power-level and on/off periods of an operating nuclear reactor core. This relative power indicator can then be used to infer fuel burnup and Pu production. This figure is taken from a conference presentation given on the apparatus described by N.S. Bowden et al. in NIM A 572 (2007) 985-998.

\subsection{Advancing the State of the Art}

To improve upon the utility of the reactor monitoring system there are two primary objectives:

1. Reduce the size of the instrument.

2. Develop surface-operation capability that does not require an overburden shield from cosmic rays.

The second of these two objectives is by far the most challenging. There is, however, a route to achieving a smaller instrument. The liquid-scintillator based instrument is roughly 2.5 meters on a side for a volume of $\sim 15 \mathrm{~m}^{3}$. The proposed alternative germanium detector-based technology could provide a factor of five reduction in size, down to a cube 1.5 meters on a side, having a volume of $\sim 3 \mathrm{~m}^{3}$. The advantage of a reduced of size has two separate (orthogonal) aspects. In the case when surface operation is not ideal or impossible due to the cosmic background rate, a smaller instrument makes finding a location with sufficient overburden more feasible. In the case when surface operation is possible, a smaller instrument of the $3 \mathrm{~m} 3$ size - could be deployed via a small, two-axel truck. However, taking advantage of this potential size reduction to the monitoring instrument first requires demonstration of a new type of radiation detector designed and built to measure a yet-to-be-seen scattering interaction: antineutrinonucleus coherent scattering. This NCMR project is focused on the development of a detector technology for measuring the antineutrino-nucleus coherent scattering interaction. Such a detector could then be used to pursue a demonstration of a reactor monitoring instrument of reduced size. 
The relevant interaction, coherent antineutrino-nucleus scattering $\left(\tilde{v}+N \rightarrow \tilde{v}^{*}+N^{*}\right)$ is predicted by the Standard Model of particle physics but has never been observed experimentally. The difficulty arises in the need for a detector having a low energy threshold and a large detector mass. The low energy detection threshold requirement is due to the recoiling nucleus having energy on the order of $0.1 \mathrm{keV}$. The large detector mass (order of kilograms) requirement is due to the small antineutrino-nucleus scattering crosssection. A large detector mass provides more material with which antineutrinos can interact, thereby increasing the signal count rate.

One route to measuring antineutrino-nucleus coherent scattering is through the extension of nuclear physics techniques using solid-state photon detectors, such as gamma-ray, X-ray, and ultraviolet (UV) detectors. Conventional gamma-ray detectors have the correct size (mass) but too high an energy threshold. UV detectors have the correct energy threshold but are too small. X-ray detectors, rather than being a happy medium, fulfill neither requirement. Thus the research challenge has been to produce a large (high-mass) detector having a low-energy threshold such that antineutrino-nucleus coherent scattering can be measured experimentally. During this project PNNL and the University of Chicago have pursued developing several germanium detectors built to simultaneously satisfy the mass and energy threshold requirements. As the electronics are continually improved (i.e., energy threshold is reduced) operation at a nuclear reactor becomes necessary. In this project, not only is reactor monitoring the goal, but indeed the reactor is the best proof-of-principle test bed with an understandable, high-intensity flux of antineutrinos.

\subsection{Technology Outlook}

The recently developed low-energy threshold germanium gamma-ray spectrometer is still the most promising candidate for making the first measurement of (anti)neutrino-nucleus coherent scattering. If this detector succeeds in measuring such as cross section it could viably compete against the liquid scintillator-based reactor antineutrino monitoring devices. A germanium-based monitoring system would likely have $1 / 5$ of the volume of a liquid scintillator-based system. It remains to be seen which system has the competitive edge with respect to operation on the surface (rather than requiring a location having cosmic-ray shielding overburden).

Continued research on reducing the electronics noise, which will thereby lower the energy threshold of the germanium detectors, is critical to achieving these objectives:

1. Making the first scientific measurement of neutrino-nucleus coherent scattering

2. Demonstrate that a compact germanium detector that can perform reactor monitoring

Rarely does particle physics research have the opportunity to have such a clear and compelling application at the conclusion of the basic research effort. We hope NCMR will continue to support this effort that can benefit both basic science and research applied to topics of national security. 


\section{Appendix A: Work completed by Task from the Statement of Work}

\section{Task 1 - Project Management}

Following Pacific Northwest National Laboratory’s project management guidelines a Project Management Plan (PMP) was developed to support the NCMR project and this PMP was submitted to the NCMR Project Monitor (Travis J. Wocelka [Travis.Wocelka@patrick.af.mil] DIA/DTT-2 Zel Technologies). Additionally, quarterly reports providing status updates were prepared (with funds spent versus budget) and submitted to the Project Monitor. This Final Report fulfills the last deliverable under the Project Management Task.

\section{Task 2 - NCMR Technical Reviews}

Both NCMR Technical Reviews (Monterey, CA in October 2008 and Las Cruces, NM in May 2009) were attended in full and presentations were made by the Principle Investigator, J. L. Orrell. The presentations on this project are recorded in the meetings' booklets.

\section{Task 3 - Preliminary Radiation Background Study at SONGS}

This was completed more much efficiently than initial planned. The background radiation measurements were made at the San Onofre Nuclear Generating Station (SONGS) throughout the first HPGe system installation, discussed in more detail in Task 5. Thus no "preliminary" background assessment was needed - it was just done in parallel with Task 5 . The background radiation data collected during the HPGe system installation provides the information needed to compare between the SONGS sub-surface location and other potential future locations. For example, a background radiation survey was performed at a number of specific places in and around the Columbia Generating Station (CGS) located at Hanford, WA (See below). The CGS reactor is an example of a reactor located fully on the surface, having no subsurface locations in which an antineutrino detection system could be placed. This is relevant to the NCMR reactor antineutrino project because any long-term viability of this technology as an applied instrument, demands that such a system be able to make the measurements at a reactor like CGS.

Sandia National Laboratory reports the measurements made at CGS in an IEEE conference record (2008 IEEE Nuclear Science Symposium Conference Record N47-2, Toward Above-ground Antineutrino Detectors for Cooperative Monitoring: Background Radiation Studies, pp. 3045-3048). This paper reports results from CGS as well as a comparison to other locations. The tabulated results are reproduced here for convenience. In addition to the tabulated average results, there were a number of conclusions about the CGS reactor's potential as a place to make reactor antineutrino measurements:

- At locations inside the reactor building, near the reactor core concrete wall, the radiation backgrounds (neutrons) were variable as a function of time

- There are locations, along the bottom of the reactor-core's concrete wall, that provide a small effective overburden against cosmic rays. This effect is a function of elevation below the top of the reactor core. That is, measurements made lower down along the concrete wall will have a greater effective overburden. All reactors have large concrete structures, therefore there will always be advantageous locations for staging a monitoring instrument in the absence of an underground location. 
- Locations outside the reactor building have more stable radiation backgrounds, but are generally further away, greatly reducing the antineutrino flux-density and, as a result, the measurement sensitivity.

In general, however, it seems very clear that without a first confirmatory measurement of the antineutrino-nucleus coherent scattering interaction at a location such as SONGS, it would be unwise to attempt such a measurement at a more difficult location such as CGS. This conclusion likely extends to all reactors where a surface-based measurement system would be required. Although these measurements at CGS performed by SNL and PNNL were not originally part of the Task 3 scope, they provide valuable information about the future challenge of bringing these antineutrino monitoring systems to the surface.

\begin{tabular}{lllll}
\hline \multicolumn{5}{c}{ ScalaR Rates } \\
\hline Deployment & Gamma & Muon & Fast & Slow \\
location & Rate & Rate & neutron & neutron \\
& {$[\mathrm{Hz}]$} & {$[\mathrm{Hz}]$} & rate $[\mathrm{Hz}]$ & rate [Hz] \\
Livermore, Ca & 93.38 & 26.7 & 0.67 & 0.035 \\
UC 2 floor $^{\text {nd }}$ & 129.69 & 25.0 & 0.1 & 0.014 \\
UC at 6 mwe & 92.36 & 13.7 & $6 \times 10^{-3}$ & $1.0 \times 10^{-3}$ \\
CGS & 50.47 & 16.9 & 0.67 & 0.043 \\
\hline
\end{tabular}

Table A-1 - Comparison of scalar rates measured at various locations including the Columbia Generating Station (CGS) as reported by SNL in a IEEE conference record (2008 IEEE Nuclear Science Symposium Conference Record N47-2, Toward Above-ground Antineutrino Detectors for Cooperative Monitoring: Background Radiation Studies, pp. 3045-3048). The gamma rate was determined by a NaI detector. The muon rate was determined by a plastic scintillating paddle. The fast neutron rate was determined by a liquid scintillation cell. The slow neutron rate was determined by a ${ }^{3} \mathrm{He}$ gas tube.

\section{Task 4 - Background Mitigation}

The Background Mitigation Task is an aggregation of methods for improving the signal-to-noise ratio of the antineutrino detection system. The method explored was driven by the reality of the status of the detection system. The initial data acquisition system for the detector (used for the work of Task 5) was based on analog amplifiers and filters. Specifically, an electronic noise rejection scheme was used whereby two analog spectroscopic amplifiers were used on each of the two detector signal outputs, but the integrating filter used by each spec. amp was set to a different value (either "fast" or "slow"). The ratio of output-signal amplitudes from the fast and slow spectroscopic amplifiers provides a basis for rejecting electronic noise. However, the method does not allow the analyst to review the original data and adjust the spectroscopic amplifier filter settings. In this way the electronic noise rejection of the analog system is inflexible.

A fully digital data acquisition system that records the digitized waveforms of the output pulses would allow for the flexibility afforded by post-data collection processing and analysis. The efforts on this front were leveraged from other projects (see the Dark Matter topic in Appendix B), but we anticipate applying the results of this effort to future work on antineutrino detection using the low-energy threshold germanium detector system. The challenge presented by using a digitizing data acquisition system is one of reaching the electronic noise threshold. To explain in contrast, an all-analog system makes a smooth transition from events above the noise threshold to those that populate the low-energy noise spike where the event rate increases exponentially. A digital system, on the other hand, is challenged to acquire and digitize all the events in the exponentially rising event rate of the noise peak at the lowest energy. In fact, most commercial off-the-shelf digitizing cards are not built with large enough on-board digital memory 
(i.e., recordable trace length) to average over baseline noise and trigger on the correct events. The first half the last year's effort was spent in discovering that our off-the-shelf components were not going to be sufficient.

A solution to this problem is to use a digitization card that was intended to handle very high event rates, such as the GRETINA digitization card developed for the GRETINA nuclear gamma-ray spectroscopy experiment which will study nuclear properties and structure. The University of Washington has one of these cards and has worked with us to understand the details of data collection using a digitizing system pushed to acquire events just below the noise threshold - an implicitly event rate limiting situation. Figure A-1 shows examples of very low-energy pulses collected by the GRETINA digitizing card. These pulses show a yet-to-be-understood feature of the low-energy threshold germanium detector. Specifically, there are apparently two populations of events: fast and slow pulse rise times. This distinguishing feature of the pulses is not available to the analyst looking at the data collected from the all-analog data acquisition system. Work has shown that the slow rise time pulses are devoid of the typical spectroscopic information one expects from a gamma-ray spectrometer, as seen in Figure A-2. This has been confirmed through conversations with the manufacturer. These fast and slow rise time pulse populations need further study in order to improve event discrimination and reduce the low-energy threshold.

In conclusion, the Background Mitigation task primarily considered one new method for rejection of noise events that contribute as a background to the low-energy portion of the collected data spectra.

Recall the present noise-event background-rejection method employs two spectroscopic amplifiers with separately set pulse shaping times. The present method takes the ratio of the results of these two spectroscopic amplifiers as a measure of the likely that the pulse is a noise event rather than a true energy deposition in the crystal. In the first year effort, this Background Mitigation task has observed the use of an all digital data acquisition system to collect and record the pulses shapes from the germanium detector. The idea is that with fully digitized data, more sophisticated off-line analysis will at least duplicate the effectiveness of the all-analog system and hopefully provide additional rejection capability. It is expected future efforts in this area will lead to a direct comparison of the all-analog to all-digital techniques so that the best may be selected for future use. 

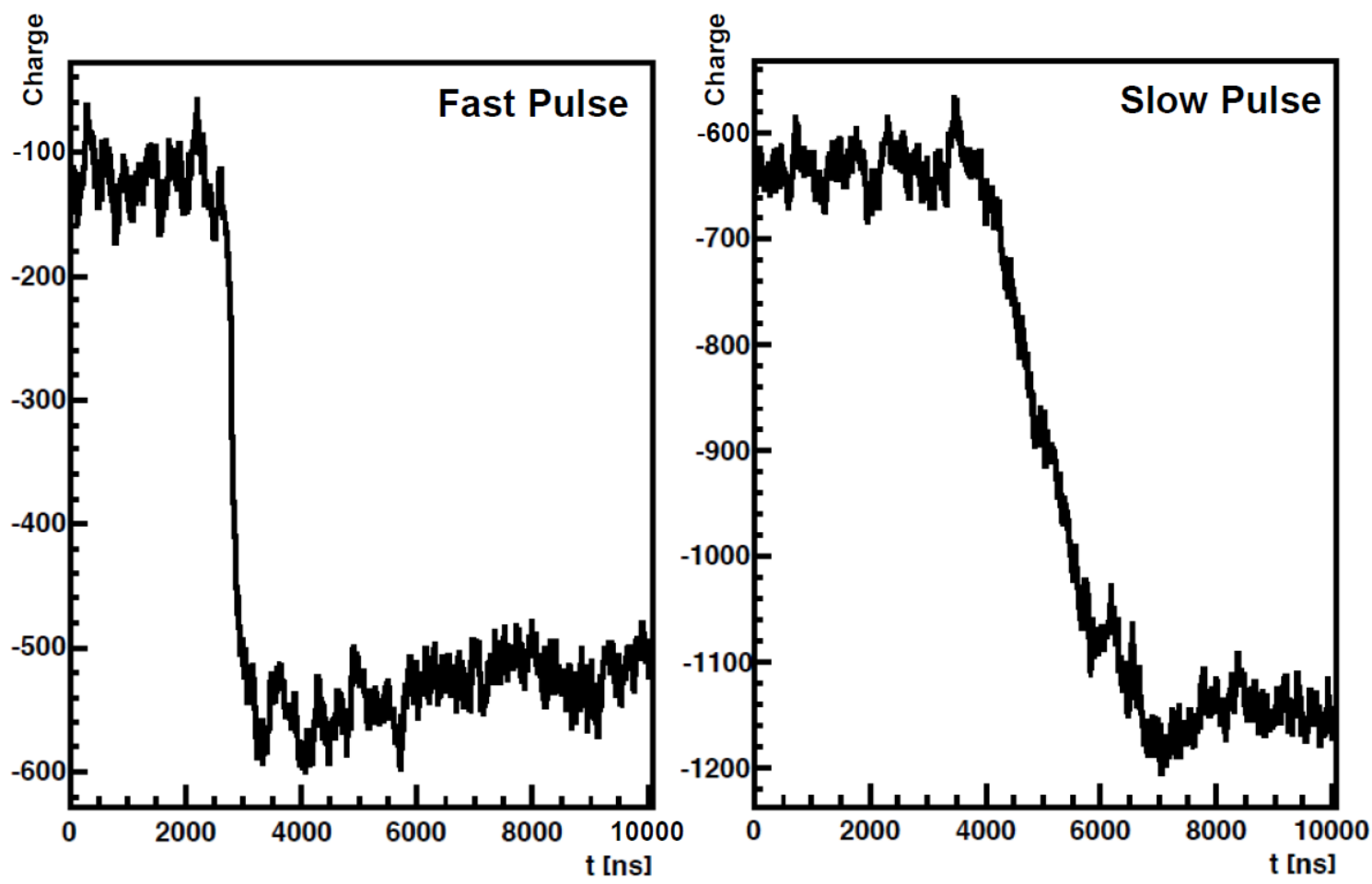

Figure A-2 - Two low-energy pulses collected by the GRETINA digitizing card. Note the pulses are actually collected - and seen here - with negative polarity (i.e. the pulses' "rising” edge goes downward as a function of time).

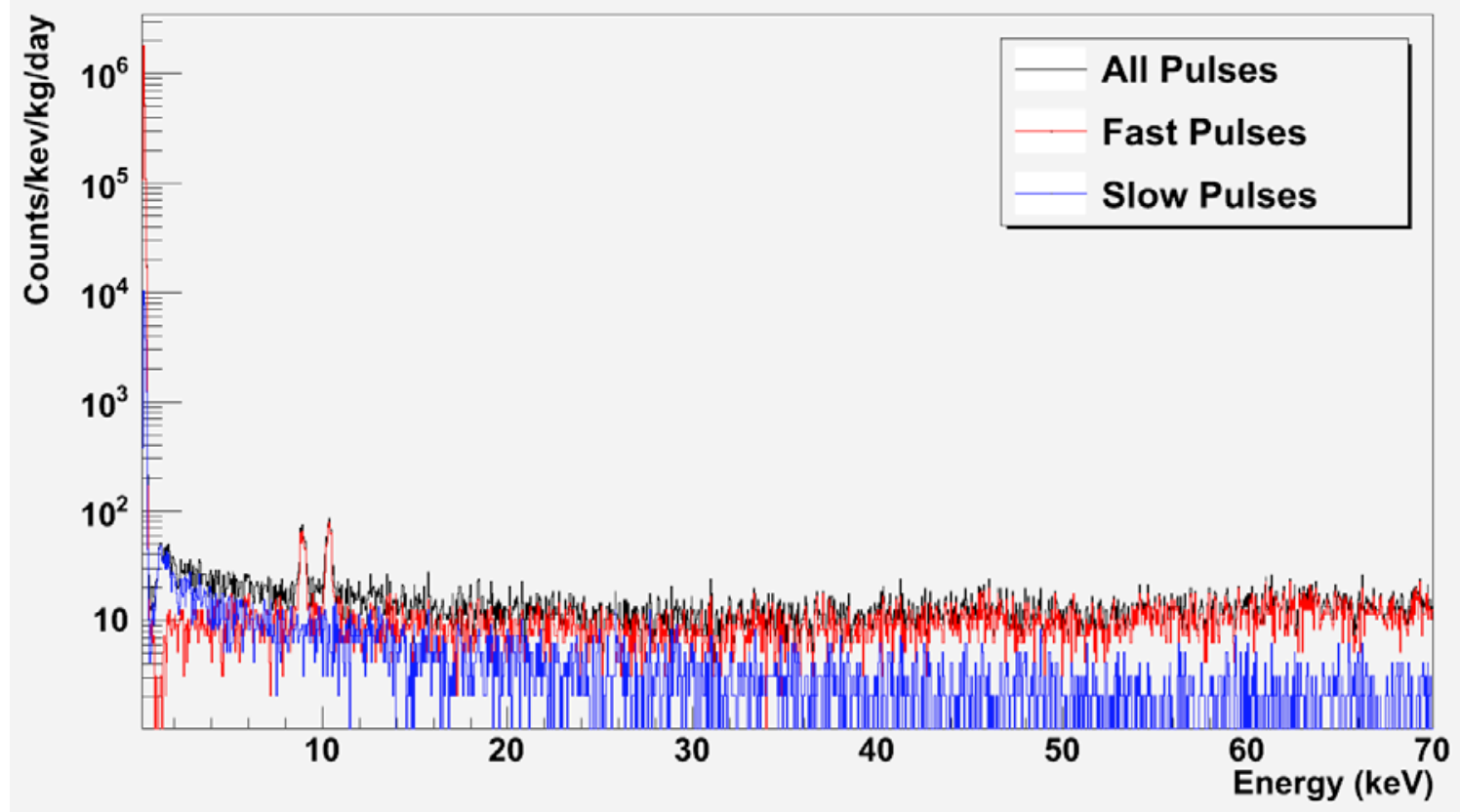

Figure A-3 - A low-energy gamma-ray spectrum taken from the Dark Matter effort described in Appendix B. The spectrum shows two populations of events - fast versus slow pulse - and how these two populations partition gamma-ray spectroscopic information from a yet-to-beunderstood (likely degraded) set of pulses. 


\section{Task 5 - SONGS HPGe System Installation}

The low-energy threshold germanium detector was installed at the SONGS in October 2008. SONGS is an ideal location to make an initial measurement of the antineutrino-nucleus coherent scattering interaction. SONGS's tendon galleries are sunk in a shallow underground location, having a roughly 30 meters water equivalent (30 mwe) overburden. This shallow overburden is ideal because it provides a shield against many cosmic rays including protons, neutrons, and mesons; however muons and atmospheric neutrinos still penetrate to this depth. In fact, the muons and neutrinos set a floor for the cosmic ray rate - only experiments located deep (thousands of feet) underground gain any appreciable shielding from muons and neutrinos. For these reasons the LLNL scintillation detector is located in these tendon galleries to facilitate demonstration of the measurement of the antineutrino flux from the reactor core. The tendon galleries are located at a radius of roughly 25 meters from the reactor core.

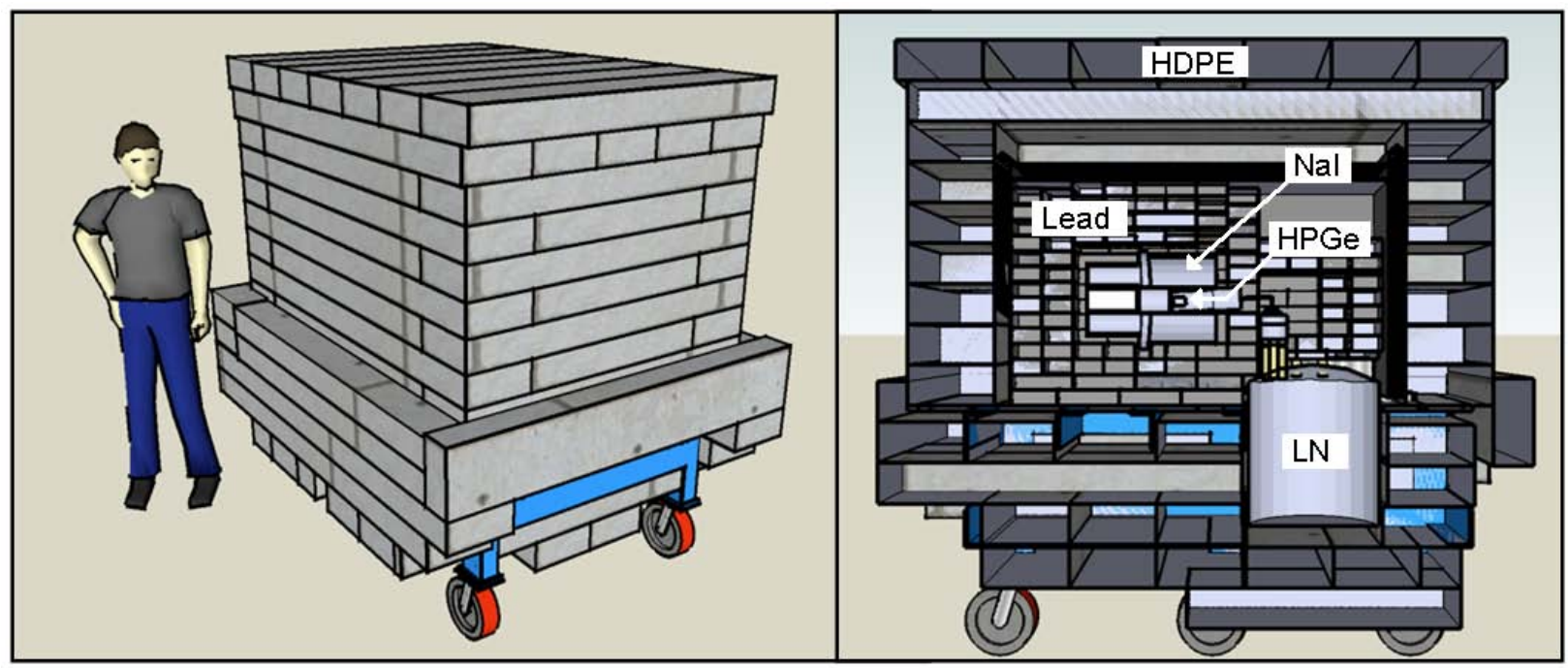

Figure A-4 - A representation of the shield design concept used at SONGS. The shield is composed of high density polyethylene (HDPE) to moderate neutrons, lead to attenuate gamma-rays, a $\mathrm{NaI}$ detector for cosmic-ray veto, and the inner-most low-energy threshold high purity germanium detector (HPGe) cooled by a liquid nitrogen (LN) bath.

Dr. Collar and his group at the University of Chicago have installed their low-energy threshold germanium detector in the SONGS tendon gallery and collected data equivalent to roughly $57 \mathrm{~kg}$ - days. The results from initial measurement phase are presented in the Task 6 description below. As of June 2009, SONGS is preparing to go into a long ( one year) down-time for overhaul maintenance and refueling of the reactor core. During this time the low-energy threshold germanium detector will be removed.

\section{Task 6 - Preliminary Signal Analysis}

This task encompassed analyzing and understanding the data collected from the installation of the lowenergy threshold germanium detector at SONGS as part of Task 5. Figure A-4 is the best summary of the data collected. 


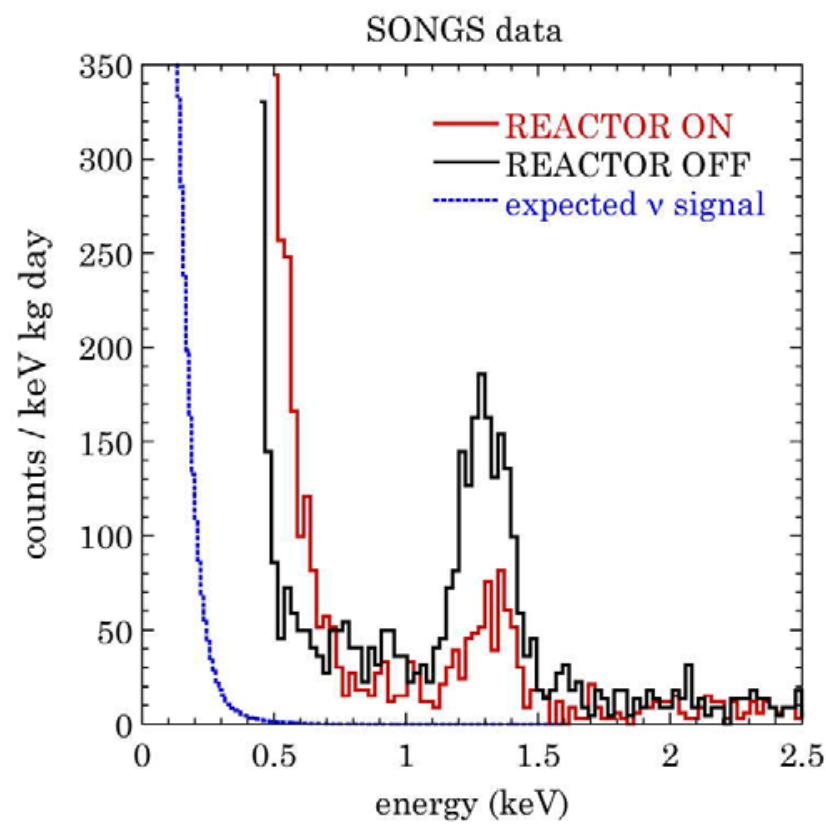

Figure A-4 - Data collected by the low-energy threshold germanium detector at the SONGS facility

Figure A-4 shows the lowest energy range $(0-2.5 \mathrm{keV})$ of the gamma-ray spectrum. To understand the figure, first note most gamma-ray spectra cover a much larger energy range spanning $0-3000 \mathrm{keV}$. There are three obvious features of the red and black spectra shown in Figure A-4. Between 1 and 1.5 $\mathrm{keV}$ there is a gallium X-ray peak. This peak is actually a result of cosmic-ray interactions and activation of the germanium crystal. Specifically, cosmic rays spallate (remove neutrons and/or protons from) germanium nuclei and in some cases generate radioactive ${ }^{68} \mathrm{Ge}$ and ${ }^{71} \mathrm{Ge}$, which then decay to gallium and result in the gallium X-ray peak seen in the figure. Next, the downward sloping background from 0.5 $2.5 \mathrm{keV}$ is the continuum background of greatest concern. This background is a relatively flat slope of events that resolve no spectroscopic features and set a limit to sensitivity to both photon (X- or gammaray) and nuclear recoils from coherent scattering. The third and final feature is the electronic noise "wall" located roughly at $0.5 \mathrm{keV}$, seen as the dramatic (nearly vertical) increase in event rate as the energy decreases.

What can we conclude from these features? The most important feature is the electronics noise "wall". This is the low-energy threshold discussed throughout the document. The onset of electronics noise is at $\sim 750 \mathrm{eV}$ and the count rate increase sharply at $\sim 500 \mathrm{eV}$. This background would hide any signal coming from the reactor antineutrinos (blue line in Figure A-4). The conclusion for the project is simply that the energy threshold needs to be lowered further to reach the neutrino signal. As a reminder, state-of-the-art $\mathrm{X}$-ray detectors routinely operate with low-energy thresholds of $50-100 \mathrm{eV}$ which, if achieved in this detector, would allow study of the anticipated neutrino signal. Going forward, achieving the $50-100 \mathrm{eV}$ energy threshold is the most important research topic.

The continuum background feature of Figure A-4 demonstrates an important achievement. For example, the Cryogenic Dark Matter Search (CDMS) located in the Soudan Mine, having 2000 mwe overburden shielding, has achieved a low-energy background continuum average on the order of $1 \mathrm{count} / \mathrm{keV} / \mathrm{kg} / \mathrm{day}$. At $2 \mathrm{keV}$, the low-energy threshold germanium detector installed with only 30 mwe shielding overburden installed near an operating nuclear reactor is shown in Figure A-4 to achieve a continuum background on 
the order of 20 counts $/ \mathrm{keV} / \mathrm{kg} /$ day. This mere factor of 20 between measured background rates is in stark contrast to the relative change is the cosmic-ray rates at the locations - roughly a factor of 25,000. Interestingly, this cosmic-ray-induced background continuum is attributed to the dead layer on the germanium crystal where the electrical contact is made. Specifically, low-energy X-rays of a few to tens of keV energy can be generated by cosmic-ray activation processes, such as described above. If those Xrays are emitted in the dead-layer of the crystal, some fraction of the energy is not collected (i.e., the "dead" part of the dead layer). These partial-charge collection events appear as a uniform continuum background, as seen in Figure A-4. In principle, it is possible to pursue a line of research that would reduce the thickness of the dead layer and there-by reduce the magnitude of the continuum background. However, this is a major undertaking and would require a serious effort on the part of the germanium detector manufacturers to re-invent their detector production process. The gallium X-ray peak is similarly a marker for the effect of cosmic-rays on the detector. This first data set collected at a reactor will serve as a benchmark for future progress with the magnitudes of both the background continuum and the gallium $\mathrm{X}$-ray peak providing quantitative measures. 


\section{Appendix B: Work leveraged from other projects}

\section{MARS R\&D: Demonstration of a field deployable $10 \mathrm{~kg}$ germanium detector array}

Multi-sensor Airborne Radiation Survey Research and Development (MARS R\&D) is a PNNL project funded by the Department of Energy National Nuclear Security Administration's NA-22 office for Nonproliferation Research \& Development. The MARS R\&D design is intended to provide 1) high relative detection efficiency ( 1000\%) needed for standoff radiation measurements, 2 ) excellent cryogenic efficiency, and 3) packaging for field deployment.

The MARS R\&D project is particularly relevant to the long-term viability of germanium-based antineutrino detection technology because it demonstrates a potential field deployment cryogenic encasement. Such a cryogenic encasement solution is a prerequisite to moving to a customer demonstration of an antineutrino measurement at a reactor. In short, the MARS R\&D project will answer the field deployment question that would normally be asked in the late 6.2 development phase (TRL 4-5). This is a significant cost savings to the full program scope required to bring a germanium-based antineutrino reactor monitor to a final customer. Said another way, once the antineutrino-nucleus coherent scattering cross-section is measured, building a suitable array of germanium detectors will be a low-risk effort, assuming success of the MARS R\&D project.
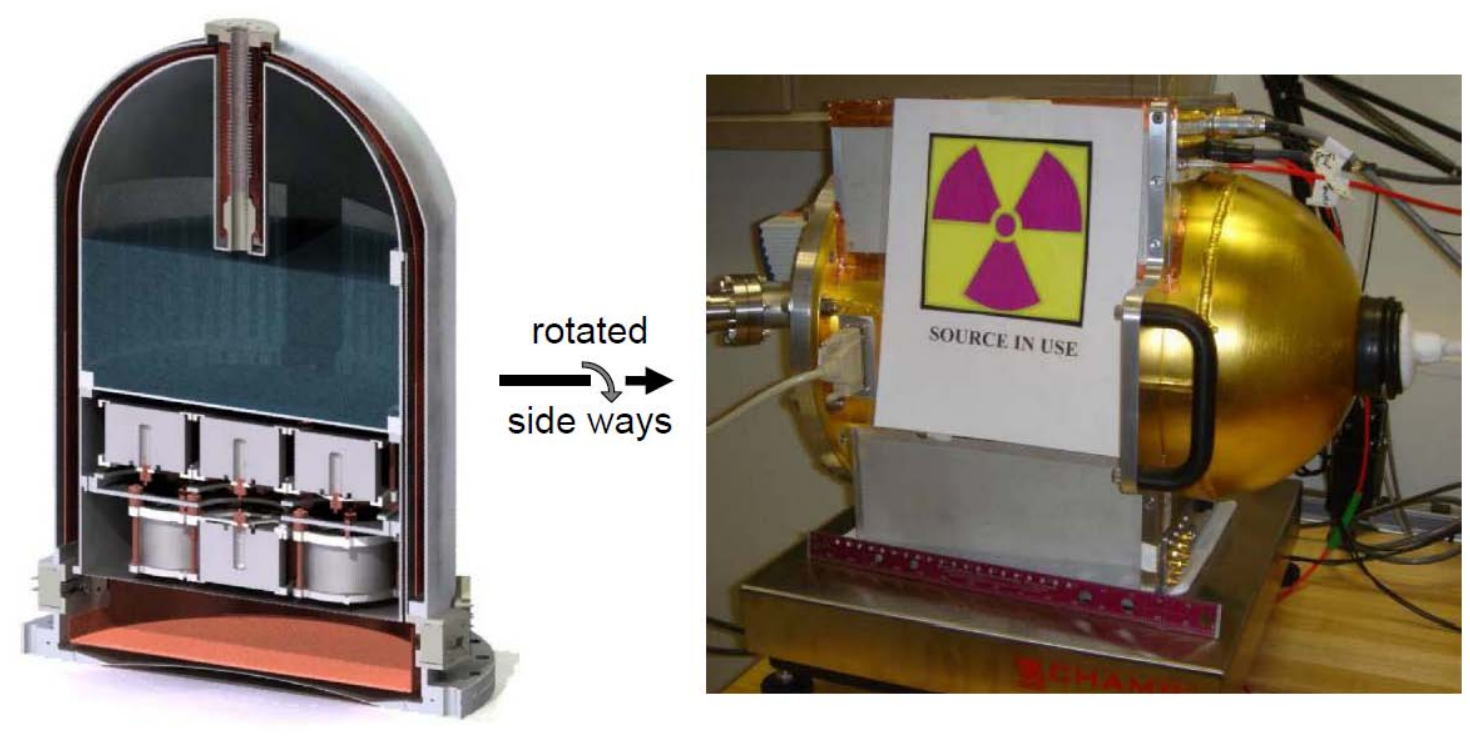

Figure B-1 - The MARS cryostat section drawing (left) and the actual MARS instrument on the test stand at PNNL

\section{Dark Matter: The same technology doing forefront science deep underground}

The low-energy threshold germanium detector design we are using to pursue neutrino-nucleus coherent scattering is also an excellent detector design for doing direct-detection searches for Dark Matter. Dark Matter is the name given to the gravitationally-active matter which, by astronomical and cosmological observations, must be present in the universe to support galaxy formation and persistence. Hypothesized Dark Matter particles can be directly detected by the recoil energy of an atomic nucleus when struck by an incoming Dark Matter particle. In the low-energy threshold germanium detector discussed throughout this report, this Dark-Matter-induced nuclear recoil has identical characteristics to those produced by an antineutrino inducing a nuclear recoil. In this way, many of the data collection and analysis techniques 
applicable to Dark Matter research can be applied equally effectively to the antineutrino-nucleus coherent scattering measurement. Two specific examples are shown by work done collaboratively with the University of Washington at the Soudan Underground Laboratory. A low-energy threshold germanium detector is installed underground in a shield (Figure B-2) with the goal of investigating Dark Matter detection techniques including fully digital data acquisition and thereby enabling pulse shape analysis for background rejection. The University of Washington is presently acquiring data using a fully digital acquisition system developed around the GRETINA digitization card. The pulse shapes collected by this method - a first for one of these detectors deployed underground - can then be analyzed offline to determine which events are true ionization events (either from nuclear recoil or scattered gamma-rays) and which are merely electronic noise blips.

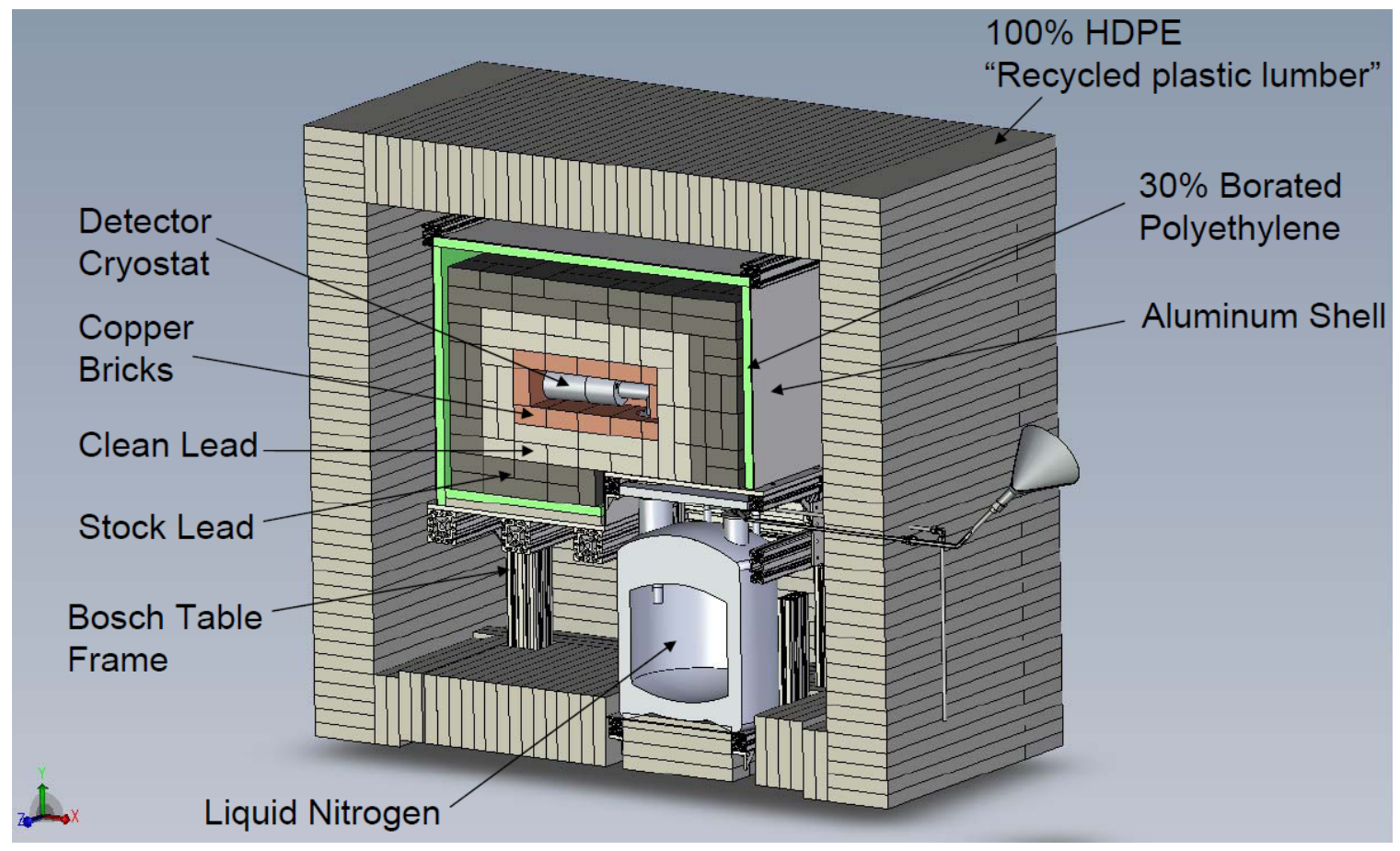

Figure B-2- Shield design for the low-energy threshold germanium detector installed at the Soudan Underground Laboratory for direct detection searches for Dark Matter 


\section{Appendix C: Program development}

Each of the following sections describes additional resources which, going forward, will contribute to the objectives of this NCMR project on reactor antineutrino monitoring.

\section{IC Postdoc}

Pacific Northwest National Laboratory has recently been awarded an Intelligence Community (IC) Postdoctoral Fellow (www.icpostdoc.com). The award is focused on the measurement of neutrino-nucleus coherent scattering, which is also the primary objective of this NCMR FY08 project. The contract is anticipated to be awarded to PNNL in June or July 2009. Tentatively, Todd W. Hossbach, who recently received a Ph.D. at the University of South Carolina, is the candidate of choice for the Fellowship position. Todd has worked at PNNL on a variety of national security-related radiation detection development projects and as well as pursuing his research on rare decays relevant to the advancement of the understanding of fundamental properties of neutrinos. These qualifications make Todd a superb choice to join Dr. Collar in Chicago for the future development of the low-energy threshold germanium detector needed to measure, for the first time, the neutrino-nucleus coherent scattering cross-section.

\section{PNNL Safeguards Summer Internship Program}

Pacific Northwest National Laboratory has an on-going Safeguards Summer Internship (SSI) Program that brings undergraduate students to the Laboratory to work on projects related to the full spectrum of nuclear safeguards issues, from policy to technology. The SSI Program pays for these students and recruits mentors from across the Laboratory to place the students in short-term safeguards related project work. In 2009 Travis Gitau, a recent Missouri University of Science and Technology graduate in nuclear engineering, will be assisting in the paper-study assessment of the detection capability of the germanium detector technology pursued by this NCMR project.

\section{Low Noise Front-end Electronics for Radiation Detectors}

Dr. Craig E. Aalseth, research scientist at Pacific Northwest National Laboratory, has tentatively agreed on a joint post-doctoral fellow who would work both on Dr. Aalseth's project and this ongoing NCMR project. Dr. Aalseth's project is developing ultra-low background, low-energy threshold proportional counters. The topic area of overlap is the focus on the electronics that enable low-energy threshold radiation detection. The very same low noise front-end amplification electronics requirements are also the major issue for the next generation of low-energy threshold germanium detectors. Lowering the germanium detector's energy threshold is the primary research issue to move forward on in the coming work on neutrino-nucleus coherent scattering. 


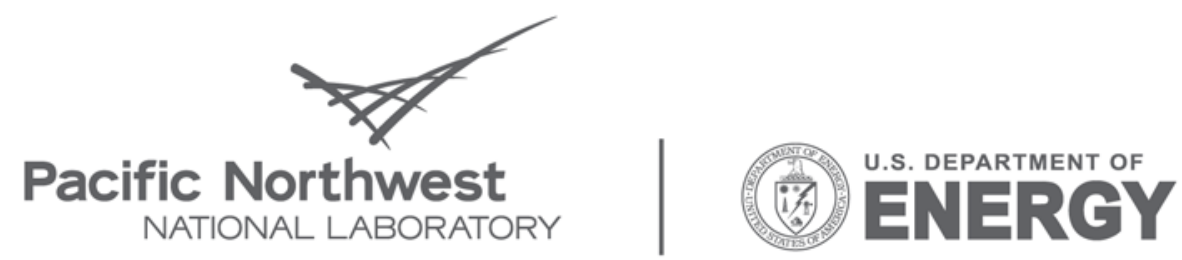

Proudly Operated by Battelle Since 1965

902 Battelle Boulevard

P.O. Box 999

Richland, WA 99352

1-888-375-PNNL (7665)

www.pnl.gov 